\title{
HST/STIS spectroscopy of the exposed white dwarf in the short-period dwarf nova EK TrA*
}

\author{
B. T. Gänsicke ${ }^{1}$, P. Szkody ${ }^{2}$, E. M. Sion ${ }^{3}$, D. W. Hoard ${ }^{4}$, S. Howell ${ }^{5}$, F. H. Cheng ${ }^{3}$, and I. Hubeny ${ }^{6}$ \\ 1 Universitäts-Sternwarte, Geismarlandstr. 11, 37083 Göttingen, Germany \\ 2 Department of Astronomy, Box 351580, University of Washington, Seattle, WA 98195, USA \\ 3 Department of Astronomy \& Astrophysics, Villanova University, Villanova, PA 19085, USA \\ 4 Cerro Tololo Inter-American Observatory, Casilla 603, La Serena, Chile \\ 5 Astrophysics Group, Planetary Science Institute, 620 North 6th Avenue, Tucson, AZ 85705, USA \\ ${ }^{6}$ Laboratory for Astronomy and Solar Physics, NASA/GSFC, Greenbelt, MD 20711, USA
}

Received 3 April 2001 / Accepted 28 May 2001

\begin{abstract}
We present high resolution Hubble Space Telescope ultraviolet spectroscopy of the dwarf nova EK TrA obtained in deep quiescence. The Space Telescope Imaging Spectrograph data reveal the broad Ly $\alpha$ absorption profile typical of a moderately cool white dwarf, overlayed by numerous broad emission lines of $\mathrm{He}$, C, N, and $\mathrm{Si}$ and by a number of narrow absorption lines, mainly of C I and Si II. Assuming a white dwarf mass in the range $0.3-1.4 M_{\odot}$ we derive $T_{\text {eff }}=17500-23400 \mathrm{~K}$ for the primary in EK TrA; $T_{\text {eff }}=18800 \mathrm{~K}$ for a canonical mass of $0.6 M_{\odot}$. From the narrow photospheric absorption lines, we measure the white dwarf rotational velocity, $v \sin i=$ $200 \pm 100 \mathrm{~km} \mathrm{~s}^{-1}$. Even though the strong contamination of the photospheric white dwarf absorption spectrum by the emission lines prevents a detailed quantitative analysis of the chemical abundances of the atmosphere, the available data suggest slightly sub-solar abundances. The high time resolution of the STIS data allows us to associate the observed ultraviolet flickering with the emission lines, possibly originating in a hot optically thin corona above the cold accretion disk.
\end{abstract}

Key words. accretion, accretion disks - stars: individual: EK TrA - stars: novae, cataclysmic variables stars: white dwarfs - ultraviolet: stars

\section{Introduction}

Photospheric emission from the accreting white dwarfs in non-magnetic cataclysmic variables $(\mathrm{CVs})$ was unmistakably identified for the first time in the quiescent ultraviolet spectra of the two dwarf novae U Gem and VW Hyi (Panek \& Holm 1984; Mateo \& Szkody 1984), obtained with the International Ultraviolet Explorer (IUE). While relatively good estimates for the effective temperatures of these stars could be derived from the IUE data, a full analysis of the properties of accreting white dwarfs in CVs - such as photospheric abundances, mass and rotation rate - had to await the availability of high resolution and high signal-tonoise ratio ultraviolet spectroscopy. A few CVs have been well studied with the Hubble Space Telescope throughout

\footnotetext{
Send offprint requests to: B. T. Gänsicke, e-mail: boris@uni-sw.gwdg.de

* Based on observations made with the NASA/ESA Hubble Space Telescope, obtained at the Space Telescope Science Institute, which is operated by the Association of Universities for Research in Astronomy, Inc., under NASA contract NAS 5-26555.
}

the first decade of its operation, demonstrating what overwhelming amount of information can be drawn from such high-quality observations, and leading to important and sometimes surprising results for our understanding of the physics of white dwarfs, accretion disks, and novae (e.g. Horne et al. 1994; Sion et al. 1994, 1996, 1997, for recent reviews see Gänsicke 2000 and Sion 1999).

However, the small number of systems studied with HST at a sufficient level of detail has thus far prevented any serious statistical analysis of the white dwarf properties in CVs and the subsequent derivation of any correlations between the observed white dwarf properties and global characteristics of these interacting binary stars, such as their orbital period or the mass transfer rate. We have initiated a large HST program, covering a total of 16 non-magnetic CVs, to significantly enlarge the observational data base, with the ultimate aim of accomplishing the first systematic study of the properties of accreting white dwarfs in CVs.

In this paper, we report the analysis of the first observations obtained in this program, unveiling the accreting white dwarf in the SU UMa type dwarf nova EK TrA. 


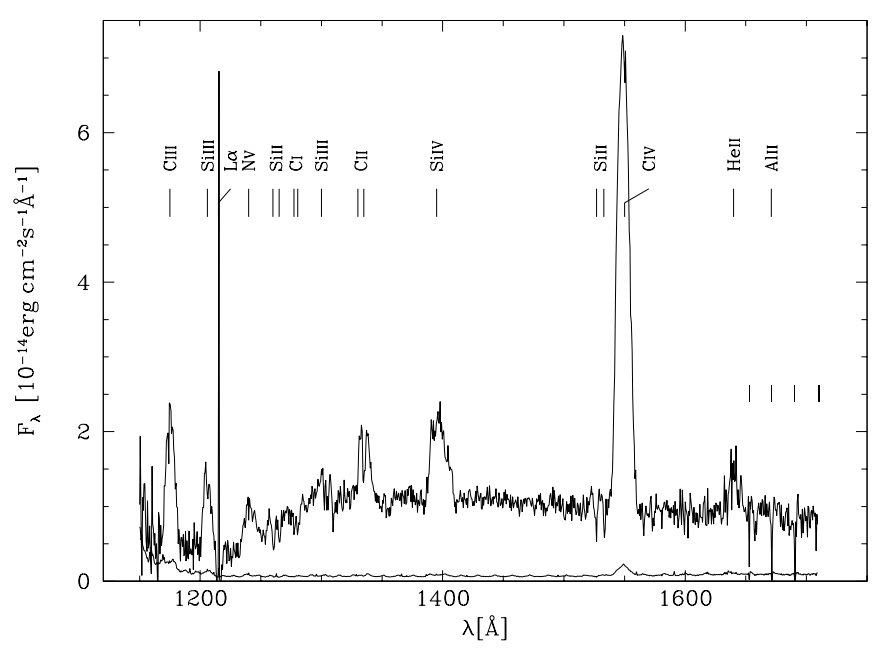

Fig. 1. The HST/STIS spectrum of EK TrA in $0.6 \AA$ bins. The line at the bottom gives the the error of the observation, which varies over each individual echelle order. The four tickmarks above the spectrum at $\lambda>1650 \AA$ show the position of the 0.4-1.0 $\AA$ wide gaps between the reddest echelle orders.

\section{Observations}

\subsection{Spectroscopy}

A single HST/STIS ultraviolet spectrum of EK TrA was obtained on 1999 July 25, UT 18:31, using the E140M echelle grating and the $0.2^{\prime \prime} \times 0.2^{\prime \prime}$ aperture, covering the range 1125-1710 $\AA$ with a nominal resolution of $R \sim$ 90000 . The choice of the echelle grating was motivated by our goal to measure the rotational velocity of the white dwarf in EK TrA, since low-resolution G140L spectroscopy can provide only a lower limit of $\sim 300 \mathrm{~km} \mathrm{~s}^{-1}$. The exposure time was $4302 \mathrm{~s}$, covering $\sim 80 \%$ of the $90.5 \mathrm{~min}$ binary orbit. Figure 1 shows the STIS spectrum of EK TrA sampled in $0.6 \AA$ bins.

The last outburst of EK TrA occured on 1999 February 20, 155 days before the HST observations. We can, therefore, assume that the STIS spectrum shows the system deep in quiescence. The continuum flux is a factor $\sim 3.5$ lower than in the faintest recorded IUE spectrum, obtained during the late decline from a super-outburst (Hassall 1985; Gänsicke et al. 1997).

Clearly visible in Fig. 1 is the broad flux turn over at $\lambda \lesssim 1320 \AA$ which we identify as the Ly $\alpha$ absorption line from the white dwarf photosphere. The signal-to-noise ratio rises from $\sim 3$ at the short wavelength end to a maximum of $\sim 15$ around $1400 \AA$ and decreases to $\sim 10$ at the long wavelength end of the spectrum. In addition to the broad Ly $\alpha$ absorption the spectrum contains a large number of medium- and high excitation emission lines, i.e. He II, CiI,III, Nv, and Si III,IV (Table 1). The narrow emission of Ly $\alpha$ is of geocoronal origin. Finally, a number of narrow $(F W H M \approx 1.5-2.5 \AA)$ absorption lines from low-ionisation transitions are observed, i.e. Si II and $\mathrm{C}_{\mathrm{I}}$ (Table 1). At the red end of the spectrum, three gaps between the echelle orders are apparent at $1653 \AA, 1671 \AA$, and $1690 \AA$.
Table 1. Line identifications in the STIS spectrum of EK TrA. $\mathrm{e}=$ emission line, $\mathrm{p}=$ photospheric absorption line, $\mathrm{i}=$ interstellar absorption line, $:=$ uncertain detection.

\begin{tabular}{rrlrrl}
\hline Ion & $\lambda[\AA]$ & Note & Ion & $\lambda[\AA]$ & Note \\
\hline C III & $1176^{*)}$ & e & Si II & $\left.1309^{*}\right)$ & $\mathrm{p}$ \\
Si III & $1207^{*)}$ & $\mathrm{e}$ & C II & $\left.1324^{*}\right)$ & $\mathrm{p}$ \\
N v & $1240^{*)}$ & $\mathrm{e}$ & C II & $1335^{*)}$ & $\mathrm{e}, \mathrm{p}, \mathrm{i}$ \\
Si II & 1260 & $\mathrm{e}, \mathrm{p}, \mathrm{i}$ & Si IV & $1400^{*)}$ & $\mathrm{e}$ \\
Si II & $1265^{*)}$ & e:, p & Si II & 1527 & $\mathrm{e}, \mathrm{p}, \mathrm{i}$ \\
C I & $1277^{*)}$ & $\mathrm{p}$ & Si II & 1533 & $\mathrm{e}, \mathrm{p}$ \\
C I & $1280^{*)}$ & $\mathrm{p}$ & C IV & $1550^{*)}$ & $\mathrm{e}$ \\
Si III & $1294-1303^{*)}$ & $\mathrm{e}$ & He II & $1640^{*)}$ & $\mathrm{e}$ \\
O I & 1302 & $\mathrm{i}$ & Al II & $1671^{+)}$ & $\mathrm{p}$ \\
Si II & $1305^{*)}$ & $\mathrm{p}, \mathrm{i}$ & & & \\
\hline
\end{tabular}

*) unresolved doublet/multiplet; ${ }^{+)}$falls near one of the gaps between the echelle orders.

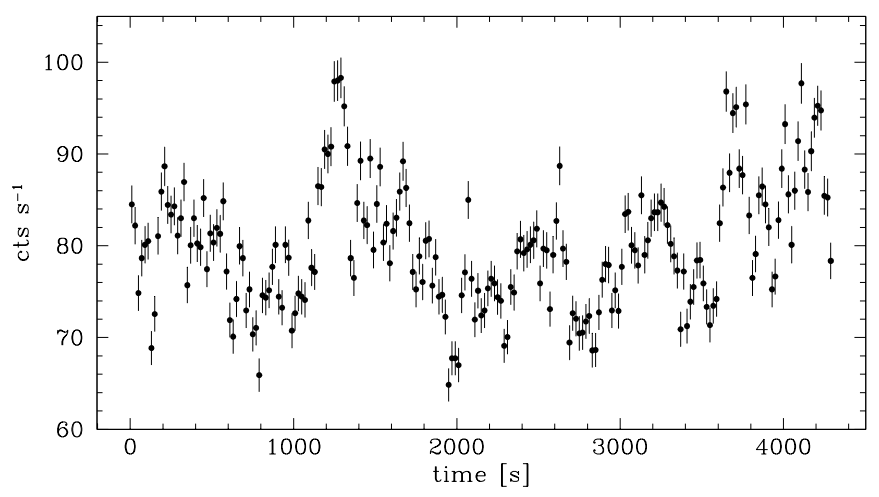

Fig. 2. STIS G140L count rate in $20 \mathrm{~s}$ bins.

\subsection{Photometry}

The STIS data were taken in the time-tagged mode, which provides information on the temporal variability of the ultraviolet flux with a $125 \mu$ s resolution. We have extracted the arrival time for all photons, excluding only a small region of the detector containing the geocoronal Ly $\alpha$ line. Figure 2 shows the resulting light curve binned to a time resolution of $20 \mathrm{~s}$.

We obtained ground-based photometry of EK TrA contemporaneous with the HST observations using the $0.9-\mathrm{m}$ telescope at Cerro Tololo Inter-American Observatory (CTIO). These observations were obtained on the night of 1999 July 25-26 UT from 23:00 to 04:00. The data consist of an $\approx 4.25 \mathrm{hr}$ long light curve of EK TrA, composed of alternating $45 \mathrm{~s}$ exposures in $V$ and $I$ (Fig. 3). In addition, we obtained observations of Landolt standard stars (Landolt 1992) and three sets of individual BVRI observations (each composed of three images in each filter) of EK TrA at the start, middle, and end of the light curve. The images were reduced in the standard fashion with IRAF tasks utilizing zero and sky flat field images obtained on the same night. We measured instrumental magnitudes using the IRAF task qphot with a 10 pixel $\left(4^{\prime \prime}\right)$ radius aperture $(\approx 4 \times$ the seeing $F W H M)$, and then 

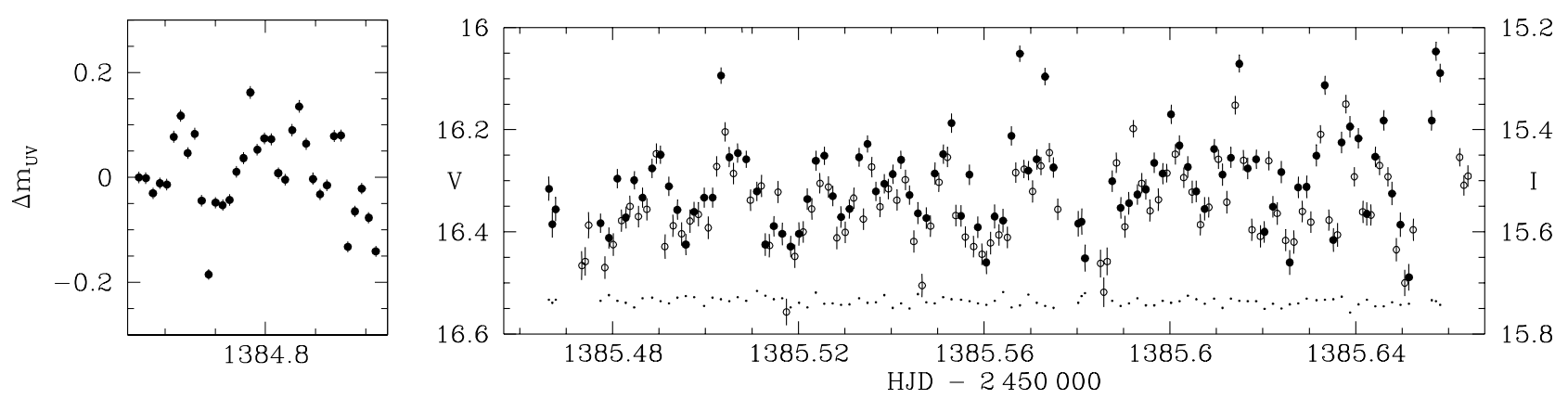

Fig. 3. Quasi-simultaneous ultraviolet (left), optical, and near-infrared photometry (right, filled symbols: $V$, open symbols: $I$ ) of EK TrA. The STIS data have been binned in $120 \mathrm{~s}$. The light curve of the comparison star used in the reduction of the ground-based data is shown as small dots (shifted down by $1.9 \mathrm{mag}$ ).

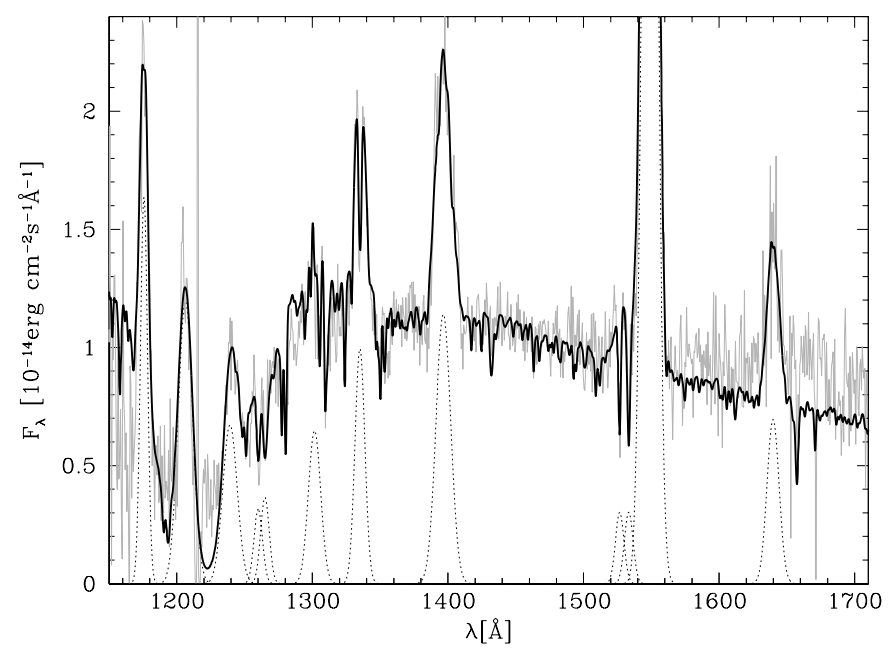

Fig. 4. The HST/STIS spectrum of EK TrA (gray line) along with a $\left(T_{\text {eff }}=18800 \mathrm{~K}, \log g=8.0\right)$ white dwarf + Gaussian emission line model. The contribution of the Gaussian emission lines is shown for clarity also by dotted lines.

calibrated them using the standard star data. The mean values and $1 \sigma$ uncertainties of the nine BVRI observations obtained for EK TrA are: $B=16.261 \pm 0.121, V=$ $16.267 \pm 0.135, R=15.854 \pm 0.076$, and $I=15.590 \pm 0.071$. The uncertainties of these mean magnitudes include both random (detection noise and flickering) and systematic (calibration) effects. Typical single measurement systematic uncertainties are $\sigma_{B}=0.035 \mathrm{mag}, \sigma_{V}=0.019 \mathrm{mag}$, $\sigma_{R}=0.014 \mathrm{mag}$, and $\sigma_{I}=0.014 \mathrm{mag}$; typical detection noise uncertainties for the $V$ and $I$ light curves are $\sigma_{V}=\sigma_{I} \lesssim 0.02 \mathrm{mag}$.

\section{Analysis}

Gänsicke et al. (1997) analysed an IUE spectrum of EK TrA obtained during the late decline from a superoutburst with a composite accretion disk plus white dwarf model. They found that a white dwarf with $T_{\text {eff }} \approx$ 16 000-20 000 contributes $\sim 25 \%$ to the ultraviolet flux. Considering that the first HST observation of EK TrA was obtained a long time after its last outburst, and that the ultraviolet flux corresponds quite well to the flux level of the white dwarf predicted by Gänsicke et al., we modelled the observed ultraviolet spectrum with a set of white dwarf model spectra, and neglect the possible continuum contribution of the accretion disk. The likely contribution of the disk is discussed below.

\subsection{White dwarf effective temperature and surface gravity}

We have computed a grid of solar abundance model spectra covering $T_{\text {eff }}=16000-20000 \mathrm{~K}$ in $200 \mathrm{~K}$ steps and $\log g=7.25-8.50$ in 0.25 steps for the analysis of the photospheric white dwarf emission. This spectral library was generated with the codes TLUSTY195 and SYNSPEC45 (Hubeny 1988; Hubeny \& Lanz 1995). We fitted the model spectra to the STIS data, allowing for Gaussian emission of He II, C II,III, Nv, and Si II,III (see Table 1), and we excluded from the fit a $20 \AA$ broad region centered on the geocoronal Ly $\alpha$ emission. In order to achieve a physically meaningful fit, we had to constrain the components of the Si II $\lambda 1260,65$ doublet to have the same $F W H M$, and the components of Si II $\lambda 1527,33$ to have the same FWHM and flux.

It is, in principle, possible to derive from such a fit both the effective temperature and the surface gravity of the white dwarf, as both parameters determine the detailed shape of the photospheric Ly $\alpha$ absorption profile. Unfortunately, in our observation of EK TrA the Ly $\alpha$ profile is strongly contaminated by various emission lines. In addition, the pressure-sensitive $\mathrm{H}_{2}^{+}$transition at $1400 \AA$, which is formed in a white dwarf photosphere with $T_{\text {eff }} \lesssim 20000 \mathrm{~K}$, is totally covered up by emission of Si IV $\lambda 1394,1403$. The best-fit parameters $\left(T_{\text {eff }}, \log g\right)$ are approximately linearly correlated, $T_{\text {eff }} \approx 2360 \times \log g-95$ with insignificant variations in $\chi^{2}$. As a result, it is not possible to derive an estimate for the surface gravity, and, hence, for the mass of the white dwarf. For the range of possible white dwarf masses, $0.3-1.4 M_{\odot}$, the fit to the STIS data constrains the white dwarf temperature to $T_{\text {eff }} \approx 17500-23400 \mathrm{~K}$, which confirms the results obtained by Gänsicke et al. (1997). The scaling factor of the model spectrum provides an estimate of the distance to EK TrA which depends, however, on the assumed white 
dwarf mass. For a typical $0.6 M_{\odot}$ white dwarf, $d=200 \mathrm{pc}$, for the extreme limits $M_{\mathrm{wd}}=0.3(1.4) M_{\odot}, d=300$ (34) pc. Considering that Gänsicke et al. (1997) derived a lower limit on the distance of $\sim 180$ pc from the nondetection of the donor star - in agreement with the $200 \mathrm{pc}$ estimated by Warner (1987) from the disk brightness - a massive white dwarf $\left(M_{\mathrm{wd}} \gtrsim 1.0 M_{\odot}\right)$ can probably be excluded.

A model fit with $\left(T_{\text {eff }}=18800 \mathrm{~K}, \log g=8.0\right)$, corresponding to a white dwarf mass of $\sim 0.6 M_{\odot}$, is shown in Fig. 4. The observed flux exceeds the model flux by $\sim 10-15 \%$ at the red end of the STIS spectrum ( $\lambda \gtrsim 1550 \AA$ ). In the optical, the model shown in Fig. 4 has $V=17.4$, which is well below the quasi-simultaneous optical magnitude of our CTIO photometry (Sect. 2.2). The optical flux excess over the white dwarf contribution has been modelled by Gänsicke et al. (1997) with emission from an optically thin accretion disk, providing a quite good fit to the Balmer emission lines. It is very likely that the flux excess at ultraviolet wavelengths is related to the same source. Indeed, the flux contribution of an optically thin accretion disk is dominated in the near-ultraviolet by bound-free opacity and its emission increases towards longer wavelengths, reaching a maximum at the Balmer jump. In view of the short available wavelength range where the disk noticeably contributes $(\sim 100 \AA)$, we refrained from a quantitative analysis of the disk contribution. Nevertheless, as a conservative estimate, the contribution of the accretion disk to the continuum flux at wavelengths shorter than $1550 \AA$ is certainly much lower than $10 \%$.

\subsection{Photospheric abundances and rotation rate}

A number of narrow photospheric absorption lines are observed that clearly have an origin in the white dwarf photosphere $^{1}$ (Table 1). Unfortunately most of these absorption lines are contaminated by optically thin radiation from the accretion disk. For instance, the STIS spectrum reveals weak emission of Si II $\lambda \lambda 1527,33$. Consequently, even though no obvious Si II $\lambda \lambda 1260,65$ emission is observed, we can not assume that the photospheric absorption lines of this Si II resonance doublet are uncontaminated. It is therefore clear that a quantitative analysis of the photospheric absorption lines will be prone to systematic uncertainties.

We computed a small grid of model spectra for $\left(T_{\text {eff }}=\right.$ $18800 \mathrm{~K}, \log g=8.0)$ covering white dwarf rotation rates of $100-500 \mathrm{~km} \mathrm{~s}^{-1}$ in steps of $100 \mathrm{~km} \mathrm{~s}^{-1}$ and abundances of $1.0,0.5$, and 0.1 times the solar values. These spectra were fitted to the strongest $\mathrm{Si}$ II and $\mathrm{C}$ I lines observed in the STIS spectrum of EK TrA, again allowing for Gaussian

\footnotetext{
1 The observed width of these lines corresponds to velocities of a few hundred $\mathrm{kms}^{-1}$. If these lines had an origin in the accretion disk, they would form in regions that are hot enough to populate Si II - i.e. in the inner disk -, where the Keplerian velocities are higher.
}

emission lines of Si II (Table 1). No emission lines were added for the $\mathrm{C}_{\mathrm{I}}$ features, as $\mathrm{C}_{\mathrm{I}}$ was - to our knowledge - never observed in emission in the ultraviolet spectra of CVs. The quality of the fits varies somewhat depending on the considered lines, but generally indicates $v \sin i=200 \pm 100 \mathrm{~km} \mathrm{~s}^{-1}$, and sub-solar abundances. The best-fit rotation rate does not significantly depend on the assumed abundances. Figure 5 shows the fits for $0.50 \times$ solar abundances and for 100,300 , and $500 \mathrm{~km} \mathrm{~s}^{-1}$.

The very narrow cores observed in SiII $\lambda 1260$ and Si II $\lambda 1527$ are of interstellar nature. Note that the $1265 \AA$ and $1533 \AA$ members of these two doublets do not show such narrow cores: they are transitions from excited levels, which are not populated in the interstellar medium. Additional strong interstellar features found in the STIS spectrum are O $\lambda$ 1302, Si II $\lambda 1304$, and $\mathrm{C}_{\text {II }} \lambda \lambda 1334,35$. All these interstellar lines are clearly visibly also in the unbinned data. Their measured positions agree within $\pm 10 \mathrm{~km} \mathrm{~s}^{-1}$ with their rest wavelengths, which nicely demonstrates the quality of the absolute wavelength calibration. The positions of the photospheric white dwarf lines constrains the systemic velocity of EKTrA to $\gamma \lesssim 70 \mathrm{~km} \mathrm{~s}^{-1}$.

\subsection{Short-term variability}

Both the ultraviolet flux (Fig. 2) and the optical flux (Fig. 3) show short-term fluctuations on time scales much shorter than the orbital period of $90.5 \mathrm{~min}\left(15.9 \mathrm{~d}^{-1}\right)$. We have computed Lomb-Scargle periodograms for the all three wavelength ranges, and find significant power at $32 \mathrm{~d}^{-1}, 67 \mathrm{~d}^{-1}, 93 \mathrm{~d}^{-1}$ in the ultraviolet and at $45 \mathrm{~d}^{-1}$ and $65 \mathrm{~d}^{-1}$ in the optical/IR. The white dwarf rotation rate derived above, $v \sin i \approx 200 \mathrm{~km} \mathrm{~s}^{-1}$, implies a spin period of a few minutes, depending somewhat on the white dwarf radius and the inclination of the system $\left(\sim 60^{\circ}\right.$, Mennickent $\&$ Arenas 1998). The detected periods are much longer, and most likely represent the preferred quasiperiodic time scale for flickering during the observations. The fact that the $V$ and the $I$ band light curves are practically identical both in shape and in amplitude is somewhat surprising, as this suggests a flat spectrum for the flickering.

In order to identify the spectral origin of the ultraviolet flickering we constructed light curves from the STIS photon event file for the strongest emission line, $\mathrm{C}$ IV $\lambda 1550$ (using the range 1530-1565 $\AA$ ), and for an adjacent emission line-free region (using the range 1414-1530 $\AA$ ). The (continuum-subtracted) C IV light curve shows a similar variation as the total light curve (Fig. 2), but with a much larger amplitude. For comparison, the standard deviation from the mean count rate is $\sigma \approx 30 \%$ in the $\mathrm{C}$ IV light curve vs. $\sigma \approx 9 \%$ in the total light curve. The variation in the continuum count rate extracted from the linefree region is $\sigma \approx 6 \%$, which is comparable to the errors due to photon statistics. This confirms the assumption that the continuum is mainly made up of photospheric emission from the white dwarf. Considering that $\sim 25 \%$ 

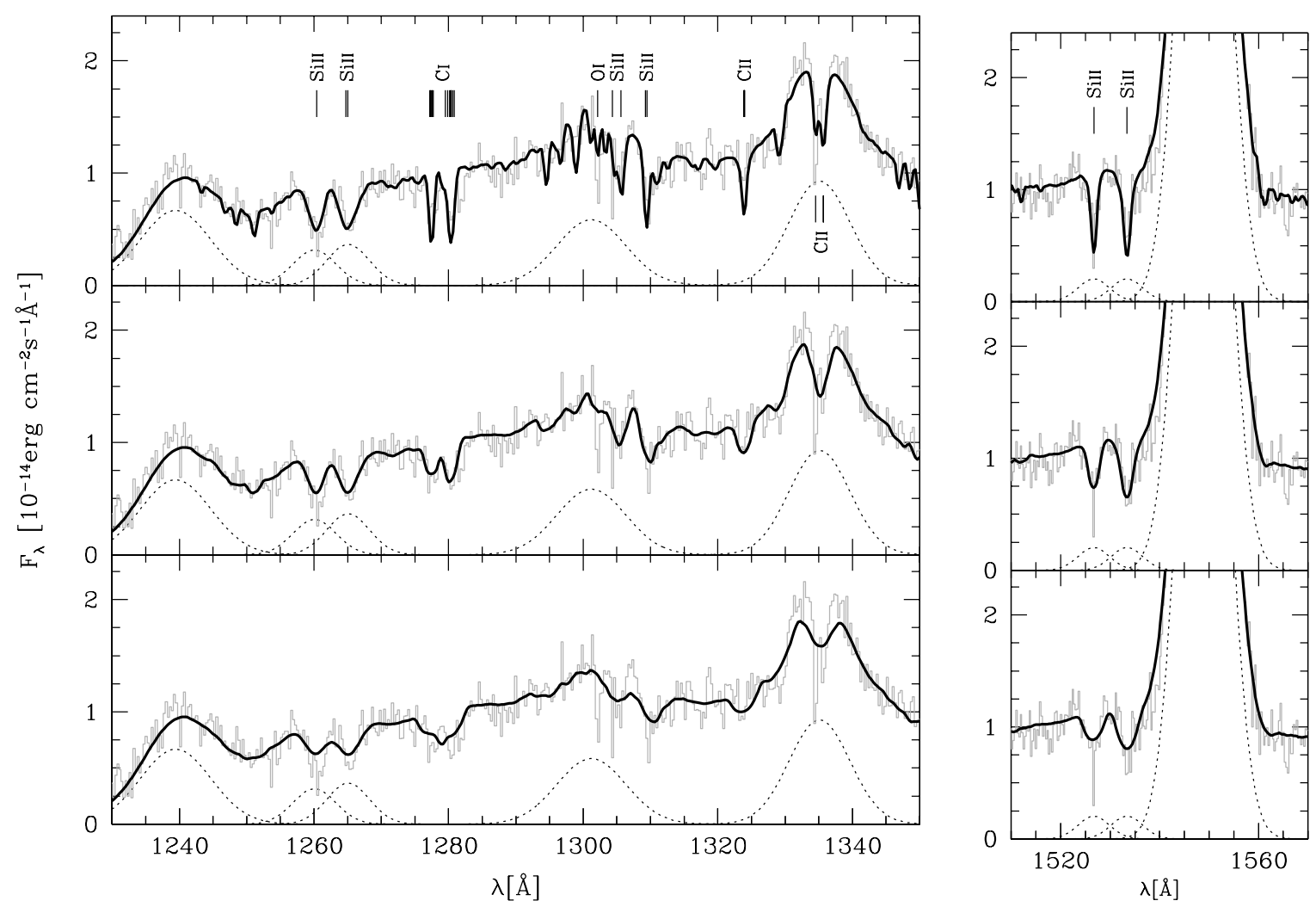

Fig. 5. Two regions of the STIS spectrum which contain narrow metal absorption lines (see Table 1). The STIS data and the model spectra are sampled in $0.3 \AA$ steps, which resolves well the observed photospheric absorption lines. Plotted as thick lines is a $\left(T_{\text {eff }}=18800 \mathrm{~K}, \log g=8.0\right.$, abundances $=0.5 \times$ solar $)$ white dwarf model, broadened for rotational velocities of 100 , $300,500 \mathrm{~km} \mathrm{~s}^{-1}$ (from top to bottom). Note the narrow interstellar absorption features at Si II $\lambda 1260$, O I $\lambda 1302$, Si II $\lambda 1304$, C II $\lambda \lambda 1334,35$, and Si II $\lambda 1527$.

of the total ultraviolet flux observed with STIS is contained in the various emission lines, we conclude that the flickering is primarily associated with an optically thin region, possibly some kind of corona above a cold accretion disk. For a discussion of the possible excitation mechanisms causing the line emission, see Mauche et al. (1997). Assuming a distance of $180 \mathrm{pc}$, the luminosity of the ultraviolet line emission is $\sim 6.6 \times 10^{30} \mathrm{erg} \mathrm{s}^{-1}$, about $\sim 20 \%$ of the sum of the optical disk luminosity and the X-ray luminosity (Gänsicke et al. 1997). Including the ultraviolet disk emission in the energy balance of the system does, therefore, not noticeably change the conclusion of Gänsicke et al. (1997) that the accretion rate in EK TrA is a factor $\sim 5$ lower than in the prototypical SU UMa dwarf nova VW Hyi.

It is interesting to compare the short-term ultraviolet variability of EK TrA with that of a long-period dwarf nova. Hoard et al. (1997) analysed fast HST/FOS spectroscopy of IP Peg and found a strong contribution of the continuum to the ultraviolet flickering. This indicates that for the higher accretion rates prevailing above the period gap the disk/corona is a significant source of ultraviolet continuum emission. Indeed, in IP Peg the white dwarf emission is not detected in the ultraviolet. Hoard et al. (1997) could also show that in IP Peg the flickering
Table 2. White dwarf rotation rates measured from Doppler-broadened absorption lines, and effective temperatures measured in deep quiescence.

\begin{tabular}{rrrrrr}
\hline System & Type & $\begin{array}{r}P_{\text {orb }} \\
{[\mathrm{min}]}\end{array}$ & $\begin{array}{r}v \sin i \\
{\left[\mathrm{~km} \mathrm{~s}^{-1}\right]}\end{array}$ & $\begin{array}{r}T_{\text {eff }} \\
{[\mathrm{K}]}\end{array}$ & Ref. \\
\hline WZ Sge & DN/WZ & 81.6 & 1200 & 14900 & 1 \\
EK TrA & DN/SU & 90.5 & 200 & 18800 & 2 \\
VW Hyi & DN/SU & 106.9 & 400 & 19000 & 3,4 \\
U Gem & DN/UG & 254.7 & $\leq 100$ & 32000 & 5,6 \\
RX And & DN/UG & 302.2 & 150 & 35000 & 7 \\
\hline
\end{tabular}

(1) Cheng et al. (1997); (2) this paper; (3) Sion et al. (1995); (4) Gänsicke \& Beuermann (1996); (5) Sion et al. (1994); (6) Long et al. (1995); (7) Sion et al. (2001).

in the continuum, medium-excitation, and high-excitation lines is uncorrelated, suggesting a rather complex multicomponent spectrum from a structured emission region.

\section{Discussion and conclusions}

EK TrA was selected as a target for our HST program as it appears to be very similar to the well-studied SU UMa dwarf nova VW Hyi (Gänsicke et al. 1997). Our analysis 
of the STIS data confirms this suggestion: we find a white dwarf temperature and rotation rate that are very close to the values derived for VW Hyi.

EK TrA is only the fifth CV white dwarf whose rotation rate could be accurately measured from the Doppler-broadened metal lines in high-resolution ultraviolet spectra, and it is only the second typical SU UMa-type system (Table 2). The other four stars include the ultrashort period large-outburst amplitude dwarf nova WZ Sge, the prototypical SU UMa dwarf nova VW Hyi, and the two long-period U Gem-type dwarf novae U Gem and RX And. If we consider the membership to one of these three groups as a measure of the evolutionary stage of a system, with the U Gem type stars above the orbital period gap being the youngest systems, the SU UMa stars below the gap being significantly older, and the WZ Sge stars being the oldest - possibly containing already degenerate donors and evolving to longer orbital periods - then the presently known white dwarf rotation rates are not in disagreement with an increase of the rotation rate with increasing age. Such a trend is indeed expected, as the angular momentum of accreted matter spins the white dwarf up (King et al. 1991), even though the long-term angular momentum evolution of accreting white dwarfs - taking into account nova explosions - is not yet well understood (Livio \& Pringle 1998).

Acknowledgements. We thank the CTIO Director's Office for the allocation of discretionary time used for this project. CTIO is operated by AURA, Inc., under cooperative agreement with the United States National Science Foundation. BTG was supported by the DLR under grant 50 OR 99036 . PS, EMS, and $\mathrm{SBH}$ acknowledge partial support of this research from HST grant GO-08103.03-97A.

\section{References}

Cheng, F. H., Sion, E. M., Szkody, P., \& Huang, M. 1997, ApJ Lett., 484, L149

Gänsicke, B. T. 2000, Rev. of Modern Astron., 13, 151

Gänsicke, B. T., \& Beuermann, K. 1996, A\&A, 309, L47

Gänsicke, B. T., Beuermann, K., \& Thomas, H. C. 1997, MNRAS, 289, 388

Hassall, B. J. M. 1985, MNRAS, 216, 335

Hoard, D. W., Baptista, R., Eracleous, M., et al. 1997, MNRAS, 288, 691

Horne, K., Marsh, T. R., Cheng, F. H., Hubeny, I., \& Lanz, T. 1994, ApJ, 426, 294

Hubeny, I. 1988, Comput., Phys., Comm., 52, 103

Hubeny, I., \& Lanz, T. 1995, ApJ, 439, 875

King, A. R., Wynn, G. A., \& Regev, O. 1991, MNRAS, 251, $30 \mathrm{P}$

Landolt, A. U. 1992, AJ, 104, 372

Livio, M., \& Pringle, J. E. 1998, ApJ, 505, 339

Long, K. S., Blair, W. P., \& Raymond, J. C. 1995, ApJ Lett., 454, L39

Mateo, M., \& Szkody, P. 1984, AJ, 89, 863

Mauche, C. W., Lee, Y. P., \& Kallman, T. R. 1997, ApJ, 477, 832

Mennickent, R. E., \& Arenas, J. 1998, PASJ, 50, 333

Panek, R. J., \& Holm, A. V. 1984, ApJ, 277, 700

Sion, E. M. 1999, PASP, 111, 532

Sion, E. M., Cheng, F., Huang, M., Hubeny, I., \& Szkody, P. 1996, ApJL, 471, L41

Sion, E. M., Cheng, F. H., Sparks, W. M., et al. 1997, ApJL, 480, L17

Sion, E. M., Huang, M., Szkody, P., \& Cheng, F. 1995, ApJL, 445, L3

Sion, E. M., Long, K. S., Szkody, P., \& Huang, M. 1994, ApJL, 430, L53

Sion, E. M., Szkody, P., Gänsicke, B., et al. 2001, ApJ, in press Warner, B. 1987, MNRAS, 227, 23 